\title{
Comunidades terapêuticas como forma de tratamento para a dependência de substâncias psicoativas
}

\author{
Therapeutic communities as an alternative to the psychoactive \\ substances dependency treatment
}

\author{
Nathalí Di Martino SABINO' \\ Sílvia de Oliveira Santos CAZENAVE²
}

\begin{abstract}
Resumo
Não há um perfil determinado para o usuário de substâncias psicoativas. Em diversas pesquisas de diferentes grupos estudados, seja pela faixa etária, gênero, nível social, econômico ou cultural, há indicação que a utilização de drogas não é restrita a um único padrão populacional. Este trabalho tem como objetivo, através de questionários respondidos por dependentes em processo de recuperação, colher informações tanto de sua vida pessoal quanto de sua dependência a substâncias psicoativas, principalmente aquelas de sua preferência, abordando como forma de tratamento as comunidades terapêuticas. A Secretaria Nacional Antidrogas vê as tais comunidades como mais uma abordagem terapêutica utilizada atualmente para o uso abusivo de drogas, juntamente com a psicoterapia analítica, terapia cognitivo-comportamental e prevenção de recaída, tornando a validação dessa metodologia importante no meio científico.
\end{abstract}

Palavras-chave: dependência de substâncias psicoativas; drogas ilícitas; comunidades terapêuticas.

\begin{abstract}
There is no determined profile for the psychoactive substances users. In various researches of different studied groups whether they're by age, gender, social, economy or cultural level, indicates that the use of drugs is not restrict to only one population pattern. Through questionnaires filled in by addicted patients in process of recovering, this work aimed at gathering information about their personal lives, as their dependency of psychoactive substances, mainly those ones of their preference, dealing with Therapeutic Community, as a way of treatment. The anti drugs national department see Therapeutic Community as one extra therapeutic approach used currently in drug abuse, together with analytic psychotherapy, cognitive-behaviorist therapy and relapse prevention, becoming important, then, the validation of this methodology in the scientific circle.
\end{abstract}

Key words: dependency of psycho active substances; drugs street; therapeutic community.

A problemática do consumo de drogas está centrada no fator da chamada "dependência". Seibel e Toscano (2001, p.137), na obra "Dependência de drogas", através de longas discussões sobre todas as questões envolvidas neste quadro, mostram que esta dependência pode ocorrer quando o uso de alguma substância psicoativa é abusivo, podendo ainda estar associada a alterações neurológicas ou psiquiátricas,

$\nabla \nabla \nabla$

1 Faculdade de Psicologia, Centro de Ciências da Vida, Pontifícia Universidade Católica de Campinas. Av. John Boyd Dunlop, s/n, Prédio Administrativo, Jd. Ipaussurama, 13060-904, Campinas, SP, Brasil. Correspondência para/Correspondence to: N.Di M. SABINO. E-mail: <nathali@terra.com.br>.

2 Faculdade de Ciências Farmacêuticas, Centro de Ciências da Vida, Pontifícia Universidade Católica de Campinas. Campinas, SP, Brasil. 
já que a maioria das substâncias provocam alterações neuropsicológicas funcionais na vigência do uso, esquematizando, assim, a ampla variedade de questões envolvidas nesse fenômeno. De acordo com o Conselho Regional de Medicina do Estado de São Paulo em conjunto com a Associação Médica Brasileira (CREMESP/AMB) (2003. p.14), "A dependência é uma relação disfuncional entre um indivíduo e seu modo de consumir uma determinada substância psicoativa", sendo vista como uma síndrome, determinada a partir de diversos fatores de risco, aparecendo em cada indivíduo de maneira distinta.

Segundo Silveira (1995), o dependente é uma pessoa que se encontra diante de uma realidade objetiva ou subjetiva insuportável, e não a conseguindo modificar ou se esquivar, resta-Ihe como única alternativa a alteração da percepção dessa realidade, que é feita pelo dependente através de substâncias psicoativas.

Por meio dessa abordagem, a dependência pode ser tratada de diversas maneiras; nesta pesquisa, destacamos o tratamento em comunidades terapêuticas (CT), principalmente devido ao seu crescimento nos últimos anos.

As informações obtidas neste estudo foram trabalhadas procurando dados que nos revelassem alguns fatores como, por exemplo: detectar drogas preferenciais, levantar motivos que levaram o paciente a dar início ao consumo de drogas e coletar o número de internações em CT e/ou outras instituições; objetivando a compreensão sobre quem é o paciente que vai à busca dessas instituições e como elas lidam com o tratamento para a dependência de substâncias psicoativas.

De acordo com Pozas (1996), as CT foram criadas em 1979 com o intuito de dar uma resposta aos problemas provenientes da dependência de drogas, possuindo assim um ambiente que necessariamente é livre das mesmas e uma forma de tratamento em que o paciente é tratado como o principal protagonista de sua cura. Trata-se de um sistema estruturado, com limites precisos e funções bem delimitadas, regras claras e afetos controlados, através de normas, horários e responsabilidades. Toda estrutura é para que o paciente se situe totalmente no tratamento, sendo assim, o trabalho intenso, tanto pela equipe profissional, quanto pelos pacientes.

Os objetivos das CT não são só os resultados do tratamento, mas também as conseqüências de uma reabilitação social envolvendo intervenção também em outros locais fora do espaço da CT (Pozas, 1996).

Hoje, para conseguir lidar com a demanda de pacientes que vão à busca de CT, a Agência Nacional de Vigilância Sanitária (ANVISA) e a Secretaria Nacional Antidrogas (SENAD) editaram uma resolução que foi capaz de regulamentar o funcionamento de todas as CT existentes no país (Agência Nacional de Vigilância Sanitária, 2001).

De acordo com Serrat (2002, p.2), membro do conselho deliberativo da Federação Brasileira de Comunidades Terapêuticas (FEBRACT) e responsável pela definição do papel das CT atualmente no Brasil, "O aumento significativo de CT é uma resposta à evolução do consumo de drogas ilícitas por parte dos jovens" e, ainda segundo o mesmo autor, quando os princípios de recuperação, resgate da cidadania, reabilitação física e psicológica e de reinserção social são corretamente aplicados, os tratamentos apresentam resultados positivos importantes, sendo o objetivo agir nos fatores psicossociais do indivíduo, ficando o tratamento medicamentoso por conta de outros órgãos, como hospitais e clínicas especializadas.

No Brasil, temos mais de 80 CT filiadas à FEBRACT. Entretanto, a preocupação maior é com as "clandestinas", para as quais a ANVISA, através da resolução RDC. no101 de 30 de maio de 2001, exigiu o funcionamento através de regulamentação, normas e fiscalização a partir de 2003, o que as submete aos conselhos de entorpecentes estaduais, municipais e do Distrito Federal e à Vigilância Sanitária.

Quanto à eficiência das $C T$, de acordo com os dados da FEBRACT, pode-se dizer que, em termos estatísticos em nível mundial, 30\% a 35\% das pessoas que freqüentaram $\mathrm{CT}$ deixaram definitivamente de consumir drogas (Serrat, 2002).

Frente às novas diretrizes estabelecidas para a regulamentação das $\mathrm{CT}$ e visando conhecer o perfil de pacientes usuários desse serviço, nos propusemos a analisá-lo através de questionários respondidos pelos próprios pacientes. 


\section{Método}

O método utilizado nesta pesquisa foi a análise qualitativa das informações; participaram cinqüenta pessoas de ambos os gêneros, que responderam ao questionário voluntariamente, sabendo que o anonimato seria preservado e que estavam participando de um evento realizado pela Febract; apenas 26 desses questionários puderam ser analisados devido ao fato de o restante não ter correspondido aos objetivos da pesquisa, já que os participantes não se declararam como dependentes ou dependentes em recuperação de substâncias psicoativas.

Quanto ao procedimento de coleta de dados, foram aplicados questionários em encontros promovidos pela FEBRACT, em participantes que estavam atuando em CT do Estado de São Paulo e que se dispuseram a colaborar com esta pesquisa. Esse questionário, elaborado pelas autoras e constituído de dez questões referentes aos objetivos propostos, visou fornecer informações gerais do paciente em recuperação, os motivos que o levaram ao uso de drogas, as substân-cias mais utilizadas e as preferenciais.

As informações colhidas das respostas aos questionários foram, a princípio, agrupadas em categorias específicas e analisadas de forma comparativa através do software Statistical Package for Social Sciences (SPSS).

\section{Resultados}

Buscando compreender quem é o paciente que busca o tratamento das CT, encontramos que o gênero dominante na pesquisa foi o masculino: 92,3\% dos questionários. A idade média foi de 36,7 anos. A maioria dos participantes é formada por solteiros e a minoria divide-se eqüitativamente entre os participantes separados e os que moram junto com o(a) companheiro(a).

O primeiro uso de drogas ilícitas ocorreu em média por volta dos 15,5 anos, sendo a menor idade encontrada de seis anos e a maior de 32 anos, indicando nesta amostra que é na fase da adolescência que geralmente ocorreu o primeiro contato com as drogas. A primeira internação teve como média a idade de 28,8 anos (Figura1).
De acordo com a Figura 2, observamos que a média de tempo em que os participantes se encontram sem fazer uso de nenhuma substância psicoativa ilícita é de 2,3 anos, tendo como período mínimo quatro meses e máximo oito anos.

O principal motivo que levou ao consumo do tóxico foi a curiosidade dos participantes, seguido da influência de amigos, sendo o menos relevante a busca de uma suposta "desinibição" (Figura 3).

As primeiras drogas usadas pelos participantes foram o álcool e a maconha; porém, as mais utilizadas pelos participantes, quando ainda eram consumidores de drogas, foram: cocaína (principalmente na forma de pasta-base, conhecida popularmente como crack), álcool, maconha, solventes, LSD, chás de lírio e cogumelos, ecstasy, heroína, anfetaminas, cigarro (tabaco), morfina, entre outras.

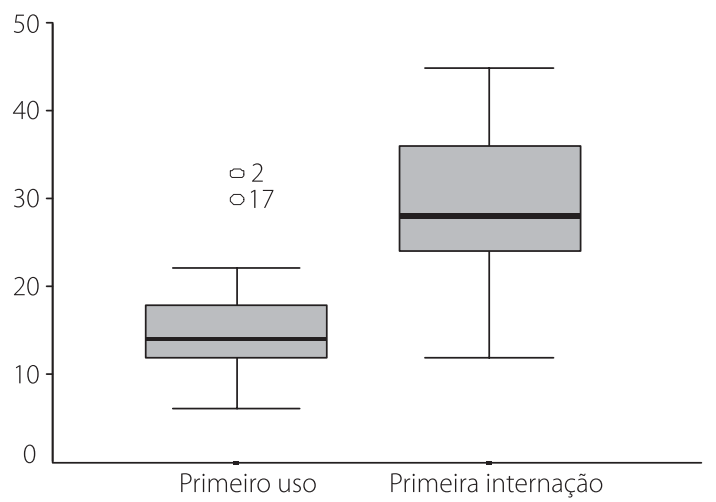

Figura 1. Idade (anos) na qual se deu o primeiro uso de substâncias psicoativas, em comparação com a idade em que ocorreu a primeira internação do participante.

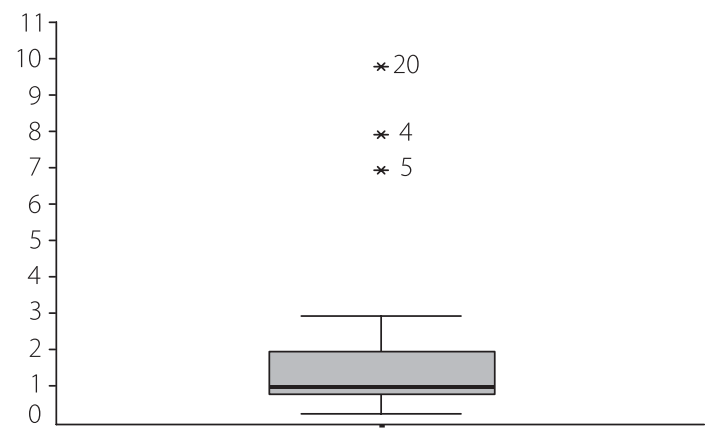

Tempo (anos) sem fazer uso de substâncias ilícitas

Figura 2. Tempo em que os participantes não fazem uso de nenhuma substância psicoativa ilícita. 


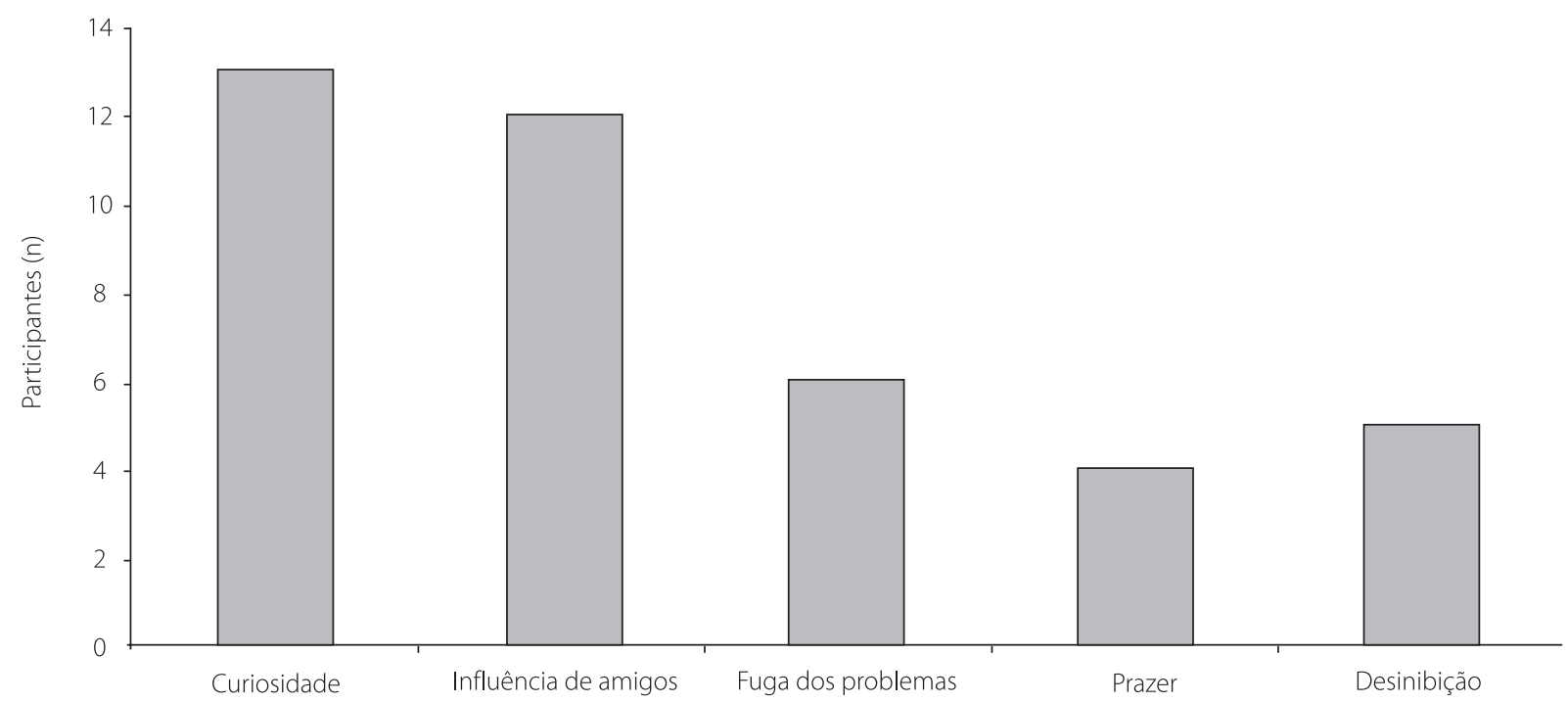

Figura 3. Motivo que levou o participante a usar a droga pela primeira vez.

OBS: Nesta questão, os participantes tinham a liberdade de escolher mais de uma opção

Questionandos sobre as internações, 50\% dos participantes já passaram por outras alternativas de recuperação em instituições como clínicas especializadas, hospitais psiquiátricos, instituições religiosas etc, além de outras comunidades terapêuticas.

\section{Discussão}

Silveira (1995) alerta que "a grande maioria dos usuários de drogas não é e nunca vai ser dependente do produto; na grande maioria das vezes, a droga é procurada como fonte de prazer tanto pelo usuário como pelo dependente; o dependente de drogas é um indivíduo para quem a droga passou a desempenhar um papel central na sua organização, na medida em que, através do prazer, ocupa lacunas importantes, tornando-se assim indispensável ao funcionamento psíquico daquele indivíduo, ou seja, um dependente, ao contrário do usuário, não pode prescindir da sua droga".

O critério utilizado pela CID-10 (Conselho Regional de Medicina do Estado de São Paulo, 2003, p.10) para definir um quadro clínico como dependência leva em conta os seguintes fatores: um forte desejo ou senso de compulsão para consumir a substância; dificuldades de controlar o comportamento de consumir a substância em termos de seu início, término e níveis de consumo; estado de abstinência fisiológico ao cessar ou reduzir o uso da substância; evidência de tolerância de tal forma que doses crescentes de substância psicoativa são requeridas para alcançar efeitos originalmente produzidos por doses mais baixas; abandono progressivo de prazeres e interesses alternativos em favor do uso da substância; e persistência no uso da substância, a despeito de evidência clara de conseqüências manifestantemente nocivas.

Nesta pesquisa não nos cabe dizer qual o grau de dependência psicológica e/ou física do paciente em recuperação. Sabemos apenas que todos os participantes necessitaram de ajuda para conseguirem interromper o uso da droga que Ihes causava dependência. De acordo com Stahl (2002, p.490), esse estágio é definido como um "estado fisiológico de neuroadaptação produzido pela administração repetida da droga, necessitando da administração para prevenir o surgimento da síndrome de abstinência"e, ainda quando se pensa no fenômeno da dependência de drogas, deve-se considerar que a exposição dos indivíduos é constante e de longo prazo. As doses variam de acordo com o usuário que procura obter um efeito agudo da substância, relacionado ao seu bem-estar. Em função desse efeito desejado é que o usuário de droga elege uma determinada substância e uma via de introdução adequada para o tempo de 
manifestação do efeito. Isso significa que quanto maior é a necessidade, maior também é a urgência de uso da droga, ou seja, quanto maior a compulsão, a via de administração escolhida será aquela de maior velocidade de distribuição pelo organismo. Deve-se considerar ainda que, dependendo da via de introdução, a droga poderá ser administrada em maior concentração, aumentando a intensidade do efeito e reforçando a compulsão (Cazenave, 1999).

Segundo a CID-10, encontramos dentro da dependência dois fenômenos também muito importantes: a tolerância ("necessidade de quantidades aumentadas da substância para atingir intoxicação ou efeito desejado") e a abstinência ("síndrome que ocorre quando se interrompe o uso do tóxico, causando malefícios que tendem a levar o dependente a querer voltar a consumir a droga").

As drogas utilizadas pelos participantes podem ser classificadas em três grupos: estimulantes: estimulam a atividade mental (anfetaminas, cocaína, crack, ecstasy, nicotina); depressores: deprimem a atividade cerebral, fazendo-os agir de maneira mais lenta (álcool, ópio, morfina, heroína); e alucinógenos/perturbadores: alteram o funcionamento cerebral, geralmente produzindo alucinações e delírios (LSD, maconha).

Os resultados desta pesquisa indicaram que as drogas mais utilizadas pelos participantes deste estudo foram a cocaína e o álcool; correspondendo perfeitamente aos resultados dos casos triados entre 1991 e 1994 pelo Programa de Orientação e Assistência a Dependentes (PROAD), nos quais as drogas mais utilizadas foram a cocaína (principalmente na forma de crack) e o álcool (Silveira, 1996).

Sobre o efeito da droga, Kalina e Kovadloff (1988, p.22) nos dizem que o dependente é sempre dominado por angústias e temores cuja intensidade os transforma em sentimentos inteiramente insuportáveis para seu ego e, "sob o efeito da droga, a sensação de fragilidade é substituída por um sentimento de extraordinária consistência e força: é a ilusão de ter conseguido superar o fracasso do ego".

Notamos que grande parte dos participantes fez uso de drogas lícitas como o álcool e o cigarro, que também possuem um alto potencial de abuso, juntamente com outras. Porém, essas não são tratadas de maneira tão profunda, devido à sua questão politicamente determinada.

O início do uso do tóxico, de acordo com esta pesquisa, ocorreu em grande parte por curiosidade, sendo seguida de uma possível influência de amigos que estimularam o uso de drogas, já que também pudemos concluir que foi na adolescência o primeiro contato com a mesma. Essa época, segundo Bee (1997, p.375), "é quase por definição, uma época de transição, uma época em que existem mudanças significativas em quase todos os aspectos da criança" e nos seus estudos sobre substâncias psicoativas nesse período da vida, o autor nos diz que "adolescentes que abusam de substâncias são altamente propensos a ter vivenciado problemas de comportamento em idades anteriores, a ter tido registros deficientes na escola, uma rejeição anterior por parte dos companheiros, negligência em suas casas, ou uma combinação de todos esses problemas, em anos iniciais de suas vidas".

Percebemos que grande parte dos participantes fez o primeiro uso por curiosidade e vontade de se desinibir; com o efeito dessas substâncias sobre o organismo, foram mais bem-recebidos pelo grupo ao qual pertenciam ou queriam pertencer, elevando assim sua auto-estima. A dissertação de mestrado de Oliveira (1997) apresentou os mesmos resultados, provando que os motivos sociais de incentivo ao início do uso de psicoativos, há sete anos, continuam sendo os mesmos nos dias de hoje.

Os participantes desta pesquisa são pessoas que estavam atuando como profissionais (sem nenhuma formação específica na área), auxiliando nas atividades da CT. O número de questionários aplicados deve-se ao pequeno acesso permitido nas próprias $C T$, e serviu como entrada preliminar para outros projetos.

Frente ao quadro atual de consumo de drogas, ocorre uma ampliação do número de instituições voltadas para o tratamento da dependência. Existem atualmente três modelos terapêuticos: internação hospitalar, que é de curta duração e, às vezes, utilizam-se medicamentos, alimentação balanceada e repouso; clínicas especializadas e/ou particulares, nas quais o tratamento é feito a médio ou a longo prazo, atendendo na maioria das vezes pacientes de alto 
poder aquisitivo; e as CT, que apresentam uma ampla flexibilidade a fim de adaptar-se a várias culturas e níveis sociais, trabalhando basicamente em três linhas de atuação: espiritual (trabalha-se com religiosidade e apoio de ex-internos), científica (psicólogos, médicos, assistentes sociais e voluntários de diversas áreas) e mista (união das duas citadas anteriormente).

O objetivo dessas instituições é o de promover uma transformação da personalidade do indivíduo, um amadurecimento pessoal e favorecer sua reinserção à sociedade. Para isso, criam-se novos valores como espiritualidade, responsabilidade, solidariedade, honestidade e amor.

As CT são um modelo de tratamento residencial utilizado durante muito tempo para pacientes com patologias psiquiátricas crônicas. Seu funciona-mento é fundamentado na premissa de que, quando não se é possível promover mudanças no indivíduo dependente, passa a ser necessário alterar a sua condição, seu meio ambiente e removê-lo da situação onde o consumo ocorre. O processo terapêutico focaliza intervenções pessoais e sociais, atribuindo funções, direitos e responsabilidades ao indivíduo dependente em ambiente seguro em relação ao consumo de drogas.

As características dessa abordagem de tratamento, segundo o Instituto Nacional sobre Abuso de Drogas dos Estados Unidos (NIDA), Lavara (2004), são "ambientes residenciais, livres de substâncias tóxicas, que usam como modelo hierárquico etapas de tratamento que refletem níveis cada vez maiores de responsabilidade social e pessoal. É utilizada a influência de companheiros para ajudar cada pessoa a aprender e assimilar as normas sociais e desenvolver habilidades cada vez mais eficazes".

De acordo com Kalina e Kovadloff (1988), o termo "comunidade terapêutica" foi primeiro usado por Maxwell Jones, que dirigia o hospital Dingleton, na Escócia. O Dr. Jones era um psiquiatra preocupado com o fato de que a psiquiatria tradicional parecia não estar ajudando os pacientes. Para solucionar esse problema, convidou vários profissionais distintos para o que chamou de "reunião mundial", visando investigar como falar diretamente com seus pacientes em seu próprio nível, procurando assim desmistificar a imagem autoritária do hospital, insistindo bastante na idéia de auto-ajuda, de ajuda mútua, de que todos deveriam trabalhar juntos para ajudarem a si mesmos e aos demais.

Segundo Baukeland (1995), a visão básica e humanitária das CT é a de que todos podem crescer e, para isso, precisam da ajuda dos outros companheiros, já que os residentes são vistos como capazes de possuir responsabilidade e, frente a isso, podem confrontar um ao outro para a obtenção de maior honestidade e maturidade.

Poulopoulos (1995) diz que as CT querem dar ao indivíduo o direito à reabilitação, em oposição às idéias de marginalização dadas àqueles que consomem drogas ilícitas.

A Senad diz que, na etapa de recuperação, é necessário que a CT se constitua em instrumento capaz de romper o vicioso círculo consumo/tratamento para grande parte dos envolvidos, tratando de forma definitiva e evitando reincidência na dependência de psicoativos.

Kalina e Kovadloff (1988) definem que os elementos centrais das CT são: 1) A CT é um elemento social. Esse sistema social deve facilitar uma aprendizagem que, em si mesmo, é terapêutica; 2) O sistema é uma ordem organizada através das normas de convivência e do enquadramento de tarefas; 3 ) O enquadramento e a normatividade baseiam-se no conceito da abstinência das drogas, enquanto se realiza o aprendizado; 4) $O$ trabalho concreto na instituição é a base da disciplina institucional e de uma educação na co-responsabilidade; 5) A CT tem hierarquias de acordo com as conquistas que cada residente tenha feito e no conhecimento de si mesmo, no trabalho sobre os outros e nas lideranças positivas que possa ir progressivamente assumindo; 6) As mudanças na instituição se concretizam através da mobilidade social, que implica a transmissão de tarefas e de responsabilidades crescentes na CT hierárquica; 7) A CT é uma sistemática de grupo, onde cada um dos grupos responde a diferentes necessidades dos residentes. Alguns seguem os alinhamentos típicos dos grupos terapêuticos. Outros terão como função atacar os comportamentos negativos para a sobrevivência do grupo e procurarão reforçar as conquistas positivas e a estrutura grupal; 8) A possibilidade de mudança mediatiza-se através da função de sustentação que tem a instituição; 9) A força terapêutica por excelência é o grupo de pares, a mudança 
precisa da interação entre os residentes. $O$ viciado recuperado integrado à equipe médica funciona como um modelo de papel útil em todo o projeto terapêutico (obs.: esses formam os participantes desta pesquisa); 10) A família está continuamente envolvida, de modo geral, em três níveis: participando da reunião semanal de terapia e da reunião mensal multifamiliar e, quando bons níveis de recuperação são alcançados, ajudando em projetos preventivos ou integrando a associação de pais, com a finalidade de trabalhar socialmente no tema da toxicomania e 11) A CT fracassará em seus propósitos se o residente não for reconhecido em sua singularidade não apenas por meio de um ato terapêutico individual, mas fundamentalmente através de uma escuta institucional de sua história de vida.

Um outro aspecto que deve ser ressaltado é a abertura das CT para outros grupos de ajuda, como Alcoólicos Anônimos (AA), Narcóticos Anônimos (NA) e Amor Exigente; o que possibilita aos internos, através das partilhas de sentimentos e situações, conhecerem as experiências pelas quais os companheiros já passaram, o que é de grande valia, além do carinho do acolhimento que os participantes desse grupo recebem e também propiciam para os outros. Dessa maneira, Fracasso (2002) diz que cada um se torna"terapeuta" de si mesmo e dos outros do grupo.

Segundo Serrat (2002), a CT é um programa muito bom, haja vista sua aplicação no mundo todo.

Porém, essa metodologia de tratamento para a dependência de substâncias psicoativas ainda requer diversos estudos para averiguar se o "isolamento" do mundo em que havia o convívio com a droga não pode ocasionar, no momento de saída da CT, uma recaída devida à desadaptação social, já que a abstinência ocorreu em um ambiente de privação tanto da substância quanto do grupo social.

Devido às $C T$ se basearem principalmente na ajuda mútua, ou seja, novos pacientes geralmente são mais auxiliados por pacientes que já conseguiram manter um determinado período sem utilizar substâncias psicoativas ilícitas, essa abordagem se torna questionável, pois não sabemos até quando a ajuda de um profissional especializado pode ser"substituída"por um outro interno, já que alguns casos exigem uma atenção direcionada e específica. Ressaltando também que este fato não ocorre em todas as instituições denominadas comunidades terapêuticas, e também não são todas que aplicam esta total substituição, já que muitas instituições possuem uma equipe multiprofissional.

As CT também devem atentar para seu objetivo de "atender pacientes dependentes de substâncias psicoativas que almejam uma recuperação", pois algumas vezes encontramos pacientes usuários ocasionais (não-dependentes), internações indiscriminadas, pacientes com outros diagnósticos psiquiátricos associados à dependência - o que requer um tratamento mais específico - e também internações utilizadas pela família do paciente com caráter punitivo.

As CT devem, seguindo as normas definidas pela Febract, definir critérios de admissão, assim como critérios de alta, atentando também para o procedimento de reinserção social do paciente.

Para um tratamento válido, os dependentes de substâncias psicoativas devem, primeiramente, acreditar que a recuperação é possível, e para isso devem contar com a experiência daqueles que estudam para lidar com essa problemática, para darem apoio e saída para que os fúteis momentos de duração do efeito de uma droga não se sobreponham àqueles de sobriedade.

\section{Conclusão}

A dependência de drogas é o desejo compulsivo de obtenção da substância, a qualquer custo, sendo uma necessidade tanto biológica (devido à neuroadaptação) quanto psicológica.

A CT é a forma de tratamento utilizada pelos participantes, tratando-se, portanto, de uma metodologia de internação em que o paciente é o principal responsável pelo seu tratamento; sendo auxiliado, porém, por companheiros do grupo, assim como por alguns profissionais da área da saúde. 0 processo terapêutico focaliza intervenções sociais, atribuindo funções, direitos e responsabilidades aos indivíduos em tratamento.

\section{Referências}

Agência Nacional de Vigilância Sanitária (2001). Resolução da Diretoria Colegiada, maio 2001 (no101). São Paulo: Anvisa/Senad. [online]. Disponível: http://www.anvisa. gov.br 
Baukeland, M. (1995). Bonding in a Therapeutic Community (pp.76-82). Grece: Kethea. (Therapy Center for dependent individuals).

Bee, H. (1997). O ciclo vital. Porto Alegre: Artmed.

Cazenave, S.O.S. (1999). Prevalência do uso de drogas na região de Campinas. Tese de doutorado, Universidade Estadual de São Paulo.

Conselho Regional de Medicina do Estado de São Paulo/ Associação Médica Brasileira. (2003). Usuários de substâncias psicoativas, abordagens, diagnóstico e tratamento. São Paulo: Cremesp/AMB.

Fracasso, L. (2002). Características da comunidade terapêutica [on-line]. Disponível: http://www. comciencia.br [Acesso: maio 2004].

Kalina, E., \& Kovadloff, S. (1988). Drogadicção. Rio de Janeiro: Francisco Alves.

Lavara, N. (2004). La eficácia de las comunidades terapêuticas a examen. [on-line]. Disponível: http:www.entorno social.es/document_n04/aass5502.html [Acesso: 7 maio 2004].

Oliveira, L.C. (1997). Variáveis críticas da reincidência às drogas em faramacodependentes. Dissertação de mestrado, Pontifícia Universidade Católica de Campinas.
Pozas, J.E.M. (1996). Comunidades terapêuticas en España: evolución histórica, situación actual y perspectivas [on-line]. Disponível em: http://www.ieanet.com/boletin/ opinion.htm

Poulopoulos, C. (1995). The new challenges for Therapeutic Comunities (pp. 103-105). Grece: kethea (Therapy Center for dependent individuals).

Seibel, S.D., \&Toscano, A. (2001). Dependência de drogas. São Paulo: Atheneu.

Serrat, S.M. (2002). Comunidades terapêuticas: mecanismo eficiente no tratamento de dependentes químicos. Entrevista [on-line]. Disponível em: http://www. comciencia.br [Acesso: 1 maio 2004].

Silveira, D.X. (1995). Drogas: uma compreensão psicodinâmica das farmacodepêndencias. São Paulo: Casa do Psicólogo.

Silveira, D.X. (1996). Dependência: compreensão e assistência às toxicomanias. São Paulo: Casa do Psicólogo.

Stahl, Stephen M. (2002). Psicofarmacologia. Rio de Janeiro: Medsi.

Recebido para publicação em 8 de setembro de 2004 e aceito em 13 de abril de 2005. 